\title{
Information Security in Cloud Computing with Elliptic Bend Cryptography
}

\author{
Vhora Arshil Nazir \\ BE (Information Technology), \\ Gandhinagar Institute of Technology, Ahmedabad, \\ Gujarat, India.
}

\begin{abstract}
Dynamic-Distributed computing is one of the present most blazing examination territories because of its capacity to decrease costs related to processing while expanding adaptability and adaptability for processing administrations. Distributed computing is Web based processing because of shared assets, programming and data are given to customers on request powerfully. Distributed computing is one of the quickest developing innovations of the IT exchange for business. Since distributed computing shares dispersed assets by means of the system in the open condition, consequently it makes security issues crucial for us to build up the distributed computing applications. Distributed computing security has become the main source of hampering its advancement. Distributed computing security has become a hotly debated issue in industry and scholastic research. This paper will investigate information security of cloud in distributed computing by executing computerized mark and encryption with elliptic bend cryptography.
\end{abstract}

Keywords: Distributed computing, Cloud Security, Information Security, Advanced Mark, Encryption, Elliptic Bend Cryptography.

\section{INTRODUCTION}

A cloud commonly contains a virtualized huge pool of processing assets, which could be reallocated to various purposes inside brief timeframe outlines. The whole procedure of mentioning and getting assets is ordinarily mechanized and is finished in minutes.

The cloud in distributed computing is the set of equipment, programming, systems, stockpiling, administrations and interfaces that joins to convey parts of figuring as an administration. Offer assets, programming and data are given to PCs and different gadgets on request. It permits individuals to do things they like to do on a PC without the requirement for them to purchase and construct an IT foundation or on the other hand to comprehend the hidden innovation.

Through cloud registering customers can get to institutionalized IT assets to send new applications, administrations or processing assets rapidly without reengineering their whole framework, henceforth making it dynamic. The center idea of distributed computing is decreasing the handling trouble on the client terminal by continually improving the taking care of capacity of the cloud. The entirety of this is accessible through a basic web association utilizing a standard program.

\section{RELATED CONCEPTS REGARDING CLOUD}

\section{Deployment Models:}

- Public cloud: the cloud framework is made accessible to the overall population individuals or a huge industry gathering furthermore, given by single specialist co-op selling cloud administrations.

- Private cloud: the cloud foundation is worked exclusively for an association. The primary bit of leeway of this model is the security, consistency and QoS.

- Community cloud: the cloud framework is shared by a few associations and supports a particular network that has shared concerns like security prerequisites, arrangement, and consistent contemplations.

- Hybrid cloud: the cloud framework is a blend of at least two mists. It empowers information application versatility through burden adjusting between mists.

\section{Characteristics Of Cloud:}

On demand service: cloud is a big useful resource and provider pool that you may get a carrier or useful esource each time you need by paying the amount that you used.

Ubiquitous community get entry to: cloud affords services everywhere even though popular terminals like mobile phones, laptops and personal digital assistants.

Easy to use: the most cloud provider's gives internet primarily based interfaces which can be easier than software application interfaces so consumers can without problems use cloud offerings.

Business model: cloud is a business version due to the fact it is pay in line with the use of carrier or useful resource.

Location impartial useful resource poling: the providers computing resources are pooled to serve more than one customers using multi tenant model with specific physical and digital assets dynamically assigned and reassigned according to call for.

\section{Cloud Solutions:}

Infrastructure as a carrier: it provides a platform virtualization surroundings as a provider in place of shopping servers, software program, facts centers.

Software as a provider: it's miles software this is deployed over internet and or is deployed to run at the back of a firewall in your LAN or PC.

Platform as a carrier: this form of cloud computing offers an improved environment as a carrier.You can use the 
middleman's device to develop your very own software and supply it to the users through internet and servers.

Storage as a provider: this is database like services billed on a software computing basis, e.g., gigabyte in step with month.

Desktop as a service: this is the provisioning of the desktop surroundings either inside a browser or as a terminal server.

\section{CLOUD SECURITY CHALLENGES}

The cloud administrations present numerous difficulties to an association. At the point when an association mitigates to devouring cloud administrations, and particularly open cloud administrations, a lot of the figuring framework foundation will now under the control of cloud specialist organization. A considerable lot of these difficulties ought to be tended to through the board activities. These administration activities will require plainly portraying the possession and duty jobs of both the cloud supplier and the association working in the job of client.

Security directors must have the option to figure out what analyst and precaution controls exist to plainly characterize the security stance of the association. Albeit legitimate security controls must be executed dependent on resource, danger, and defenselessness hazard appraisal frameworks. Distributed computing security hazard appraisal report basically from the merchant's point of view about security abilities investigated security dangers looked by the cloud. Here is the security dangers list. Administrative consistency: distributed computing suppliers who decline to outer reviews and security confirmation.

- Special clients get to: touchy information handled outside the association carries with it an inalienable degree of hazard.

- Information area: when you use cloud, you most likely won't know precisely where your information is facilitated.

- Information isolation: information in the cloud is shared condition close by information from different clients.

- Recuperation: regardless of whether you don't have a clue where your information is, a cloud supplier should mention to you what will happen to your information and administration if there should arise an occurrence of a calamity.

- Insightful help: exploring improper or criminal behavior might be outlandish in distributed computing.

- Long haul reasonability: you should be certain your information will stay accessible significantly after such an occasion.

\section{PROPOSED SECURITY SOLUTIONS}

Distributed computing is a virtual situation that requires moving information all through the cloud. In this way, a few information capacity concerns can emerge.
Normally, clients will know not one or the other the specific area of their information nor different wellsprings of the information on the whole put away with theirs.

To protect the security of your cloud-based virtual foundation, perform security best practice at both the conventional IT and virtual cloud. To guarantee information classification, verification, trustworthiness, and accessibility, the supplier ought to incorporate the accompanying:

- Encryption: the affectability of information may require that the arrange traffic to and from the virtual machine be encoded, utilizing encryption at the host operating system programming.

- Physical security: keep the virtual framework and cloud the executives has protected and secure behind checked entryways, what's more, naturally protected.

- Confirmation and access control: the verification capacities inside your virtual framework should duplicate the way your other physical frameworks verify. Once secret word and biometrics should all be executed in the same way. Additionally confirmation requires while you are sending information or message from one cloud to another cloud. To give message confirmation we will utilize computerized marks.

- Partition of obligations: as framework gets progressively perplexing, misconfiguration happens, on the grounds that absence of skill combined with lacking correspondence. Make certain to uphold least benefits with get to controls and responsibility.

- Setup, change control, and fix the board: this is significant and here and there neglected in littler associations. Setup, change control, fix the board, and refreshed procedures should be kept up in the virtual world just as physical world.

- Interruption recognition and anticipation: what's coming into and leaving your system needs to know. A host based interruption avoidance framework combined with a hypervisor based arrangement could look at for virtual system traffic.

Among these proposed security arrangements, we consider in this paper validation and encryption for secure information transmission from one cloud to other cloud that requires secure and validated information with elliptic bend cryptography.

\section{ELLIPTICAL CURVES IN CRYPTOGRAPHY}

Elliptic Bend (EC) frameworks as applied to cryptography were first proposed in 1985 freely by Neal Koblitz what's more, Victor Mill operator. An elliptic bend over a field $\mathrm{K}$ is a nonsingular cubic bend in two factors, $f(x, y)=0$ with a discerning point (which might be a point at unendingly).

The field $\mathrm{K}$ is typically taken to be the unpredictable numbers, reals, rationals, and arithmetical expansions of rationals, p-adic numbers, or a limited field. Elliptic 
bends bunches for cryptography are inspected with the hidden fields of Fp.

$$
y^{2}=x^{3}+a x+b
$$

(where $\mathrm{p}>3$ is a prime) and $\mathrm{F} 2 \mathrm{~m}$ (a twofold portrayal with $2 \mathrm{~m}$ components). An elliptic bend is a plane bend characterized by a condition of the structure think about elliptic bend

$$
E: y^{2}=x^{3}-x+1
$$

On the off chance that $\mathrm{P} 1$ and $\mathrm{P} 2$ are on $\mathrm{E}$, we can characterize expansion $\mathrm{P} 3=\mathrm{P} 1+\mathrm{P} 2$

As appeared in the picture.Let $\mathrm{P} 1=(\mathrm{x} 1, \mathrm{y} 1), \mathrm{P} 2=$ $(\mathrm{x} 2, \mathrm{y} 2), \mathrm{P} 3$

$=(\mathrm{x} 3, \mathrm{y} 3)$ and $\mathrm{P} 1$ not equivalent to $\mathrm{P} 2$.

$$
\mathrm{m}=\frac{y 2-y 1}{x 2-x 1}
$$

To discover the crossing point with $\mathrm{E}$ we get $(\mathrm{m}(\mathrm{x}-\mathrm{x} 1)+$ $\mathrm{y} 1)^{2}=\mathrm{x}^{3}+\mathrm{Ax}+\mathrm{B}$ or, $0=\mathrm{x}^{3}-\mathrm{m}^{2} \mathrm{x}^{2}+\ldots$

So, $\mathrm{x} 3=\mathrm{m}^{2}-\mathrm{x}_{1}-\mathrm{x}_{2}$

$\Rightarrow \mathrm{y}_{3}=\mathrm{m}\left(\mathrm{x}_{1}-\mathrm{x}_{2}\right)-\mathrm{y}_{1}$

Augmentation is characterized as rehashed expansion, for instance: $3 \mathrm{P}=\mathrm{P}+\mathrm{P}+\mathrm{P}$

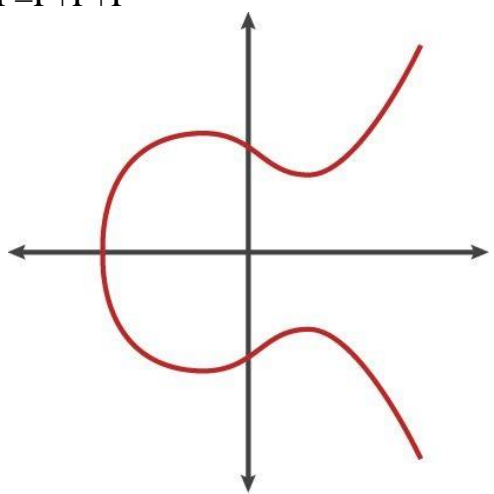

Elliptic bend cryptography [ECC] is an open key cryptosystem. Each client has an open and a private key. Open key is utilized for encryption/signature check. Private Key is utilized for decoding/signature age. Elliptic bends are utilized as an augmentation to other current cryptosystems. That is Elliptic Bend DiffieHellman Key Trade and Elliptic Bend Computerized Mark Calculation.

\section{PROPOSED PROCEDURE TO ENHANCE DATA SECURITY IN CLOUD}

Let us expect we have two associations A and B. A and B go about as open mists with information, programming and applications. A need to send information to B's cloud safely and information ought to be verified. We are here attempting to send a safe information from $\mathrm{A}$ to $\mathrm{B}$ by applying advanced mark and encryption to information with elliptic bend cryptography.
Assume B needs a XML archive from A's cloud at that point B's client will put a solicitation to A's client. A's client select comparing XML archive from A's cloud information stockpiling and afterward apply the hash work, it will give message digest. Sign the message digest with his private key by utilizing A's product. It is called computerized signature.

Scramble carefully marked mark with B's open key utilizing ECC calculation. Scrambled figure messages will be sent toB's product unscramble the figure message to XML report with his private key and confirm the mark with An's open key

\section{PROPOSED ALGORITHM FOR DATA SECURITY USING ECC}

The two mists consent to some openly known information thing

1. The elliptic bend condition

a. estimations of $a$ and $b$

b. prime, $\mathrm{p}$

2. The elliptic gathering registered from the elliptic bend condition

3. A base point, B, taken from the elliptic gathering

\section{Key generation:}

1. A chooses a whole number dA. This is A's private key.

2. A at that point creates an open key $P A=d A * B$

3. B also chooses a private key $\mathrm{dB}$ and processes a open key $\mathrm{PB}=\mathrm{dB} * \mathrm{~B}$

4. A produces the security key $\mathrm{K}=\mathrm{dA} * \mathrm{~PB}$. B creates the emit key $\mathrm{K}=\mathrm{dB} * \mathrm{PA}$

\section{Signature generation:}

1. Ascertain $\mathrm{e}=\mathrm{HASH}(\mathrm{m})$, where $\mathrm{HASH}$ is a cryptographic hash work, for example, SHA-1

2. Select an arbitrary whole number $\mathrm{k}$ from $[1, \mathrm{n}-1]$

3. Figure $r=x 1(\bmod n)$, where $(x 1, y 1)=k * B$. In the event that $r=0$, go to stage 2

4. Figure $s=k-1(e+d A r)(\bmod n)$. In the event that $s$ $=0$, go to stage 2

5. The mark is the pair $(\mathrm{r}, \mathrm{s})$

6. Send signature $(r, s)$ to B cloud.

\section{Encryption algorithm:}

Assume A needs to send to B a scrambled message.

1. A takes plaintext message $\mathrm{M}$, and encodes it onto a point, PM, from the elliptic gathering.

2. A picks another arbitrary whole number, $\mathrm{k}$ from the interim [1, p-1]

3. The figure content is a couple of focuses $\mathrm{PC}=$ [ $(\mathrm{kB}),(\mathrm{PM}+\mathrm{kPB})]$

4. Send ciphertext PC to cloud B.

\section{Decryption algorithm:}

Cloud B will find a way to unscramble figure content PC.

1. B figures the result of the principal point from PC 
and his private key, $\mathrm{dB} \mathrm{dB} *(\mathrm{kB})$

2. $\quad \mathrm{B}$ at that point takes this item and subtracts it from the second point from $\mathrm{PC}(\mathrm{PM}+\mathrm{kPB})-[\mathrm{dB}(\mathrm{kB})]=$ $\mathrm{PM}+\mathrm{k}(\mathrm{dBB})-\mathrm{dB}(\mathrm{kB})=\mathrm{PM}$

3. B cloud at that point deciphers PM to get the message, $\mathrm{M}$

\section{Signature Verification:}

For B to verify Al's mark, B must have A's open key PA

1. Check that $r$ and $s$ are whole numbers in $[1, n-1]$. If not, the mark is invalid

2. Ascertain $\mathrm{e}=\mathrm{HASH}(\mathrm{m})$, where HASH is the equivalent work utilized in the mark age

3. Figure $w=s-1(\bmod n)$

4. Figure $u 1=e w(\bmod n)$ and $u 2=r w(\bmod n)$

5. Calculate $(\mathrm{x} 1, \mathrm{y} 1)=\mathrm{u} 1 \mathrm{~B}+\mathrm{u} 2 \mathrm{PA}$

6. The mark is legitimate if $x 1=r(\bmod n)$, invalid in any case

\section{CONCLUSION}

Presently a day's distributed computing confronting numerous security challenges. Clients put their information in the cloud and move from one cloud to another, the security of clients in danger result from last control of information. Clients generally worry about information security, so virtualization security and information security are the fundamental issue of distributed computing security. We worry here about information security with Elliptic bend cryptography to give privacy and confirmation of information between mists. In future we will concern greater security issues of cloud registering and attempt to discover better arrangements utilizing cryptography.

\section{REFERENCES}

[1] Liu Peng, the definition and characteristics of cloud computing, http://blog.sina.com.cn/s/blog_5f0da5590100cmxw.html http://www.chinacloud.cn, March 9, 2009.

[2] Ya-Qin Zhang, the future of computing in the "cloud - Client", The Economic Observer reported, http://www.sina.com.cn, 2008 Nian 07 Yue 12 Ri 14:30.

[3] Jianfeng Yang and Zhibin Chen "Cloud Computing Research and Security Issues".

[4] D. L. Ponemon, "Security of Cloud Computing Users," 2010.

[5] C.Almond, "A Practical Guide to Cloud Computing Security," 27 August 20092009.

[6] IBM, "Google and IBM Announced University Initiative to Address Internet-Scale Computing Challenges," http://www03.ibm.com/press/us/en/pressrelease/22414.wss.

[7] http://en.wikipedia.org/wiki/Cloud_computing.

[8] http://www.cloudcomputing china.cn/Article/luilan/200909/306.html.

[9] http://searchcloudcomputing.techtarget.com/sDefinition/ $/ 0$, sid2 01_gci1287881,00.html.

[10] http://www.boingboing.net/2009/09/02/cloud-computing-skep. html

[11] Google, "http://code.google.com/appengine/"

[12] http://cloudsecurity.trendmicro.com/ 